\title{
Design of a Fire alarm system
}

\author{
Yuhui Cheng *, Tianyi Zhang \\ Tianjin University of Science and Technology, China; \\ E-mail: *13642017570@163.com \\ www.tust.edu.cn
}

\begin{abstract}
This article introduces the design of the fire alarm system and analyzes its working principle. The system uses a distributed sensor network based on ZigBee to achieve fire alarm and real-time evacuation. Select the infrared flame sensor and MQ2 smoke sensor as the information source. The core control board is the Arduino Mage2560 board. Through the ESP8266 module and the GPRS module, the multi-channel information transmission function can be realized. The alarm can be classified according to the fire situation.
\end{abstract}

Keywords: ZigBee, Hierarchical alarm, crowd evacuation

\section{Introduction}

With the development of cities, the increasing number of high-rise buildings has led to an increase in fire hazards. Due to the difficulty of evacuation and fire fighting in high-rise buildings, once a fire occurs, it will cause serious losses. In this case, it is particularly important to call the police in time and evacuate the crowd. ${ }^{1}$.

At present, most fire alarm devices are independent alarms. The wiring is cumbersome and the wiring is prone to aging and wear. Therefore, the probability of failure or false alarms of such alarm devices is relatively high. On the other hand, a system that only warns but does not provide specific fire conditions, nor can it guide people to evacuate.

Based on the above discussion ${ }^{2}$, we designed a new type of fire alarm system. The system obtains data through the infrared sensor and MQ2 smoke sensor and uploads it to the client through the ESP8266WIFI module. The Zigbee protocol is used for wireless communication between monitoring nodes. When a fire breaks out, the system will alarm at different levels, and guide the crowd based on the location and scale of the fire. The overall structure of the system is shown in Fig.1.

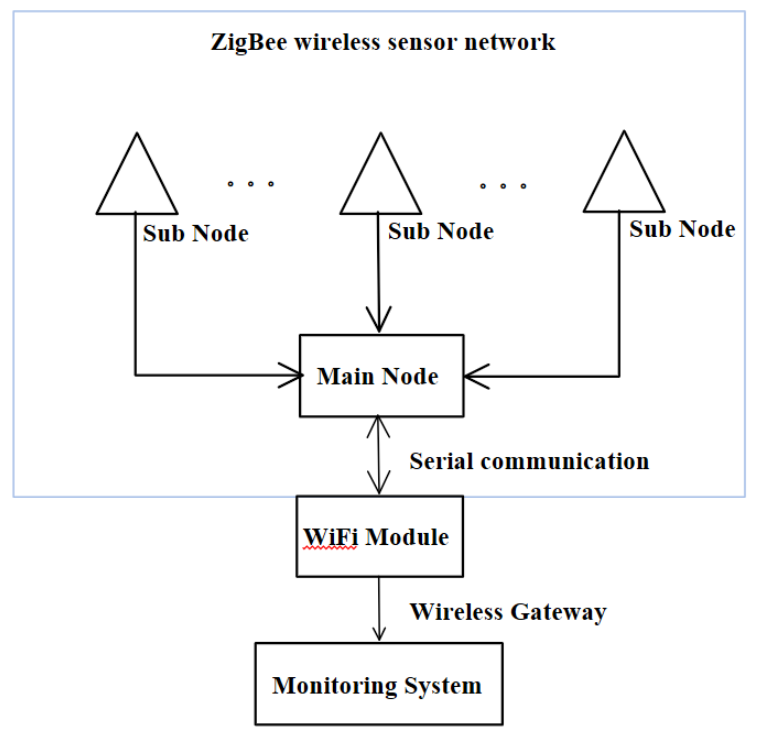

Fig.1 System structure

\section{The Hardware Structure}

The hardware of this system mainly has four parts. The Arduino Mage 2560 board and the Arduino Nano board constitute the control unit of the system node; the infrared 
sensor and the MQ2 smoke sensor on the node constitute the data monitoring unit; the CC2530 communication module, ESP8266 WiFi module and GPRS SIM300C module constitute the communication unit; the multi-directional indicator light and the voice module constitute the evacuation unit.

\section{1. control unit}

The system is composed of the main node and a sub-node. The main node uses the Arduino Mage2560 development board as the control board, and the sub-nodes are designed based on the Arduino Nano development board.

The Arduino Mega 2560 is a microcontroller board based on the ATmega2560. It has 54 digital input/output pins (of which 15 can be used as PWM outputs), 16 analog inputs, 4 UARTs (hardware serial ports), a 16 $\mathrm{MHz}$ crystal oscillator, a USB connection, a power jack, an ICSP header, and a reset button.

The Arduino Mega 2560 board is shown in Fig. 2.

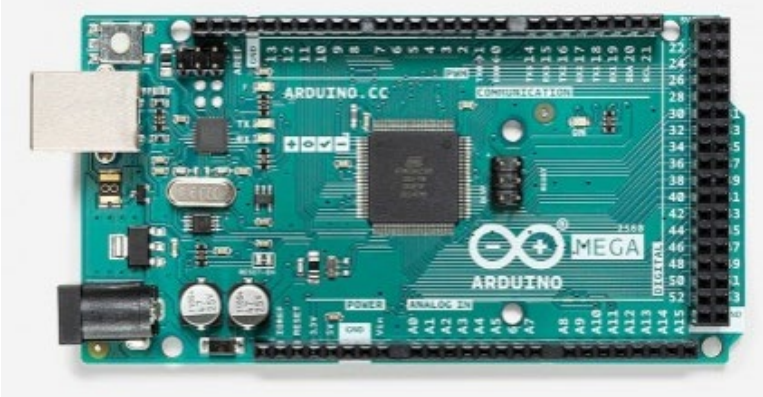

Fig.2 Arduino Mega 2560 board

The Arduino Nano is a small and complete board based on the ATmega328.

The Arduino Nano board is shown in Fig.3.

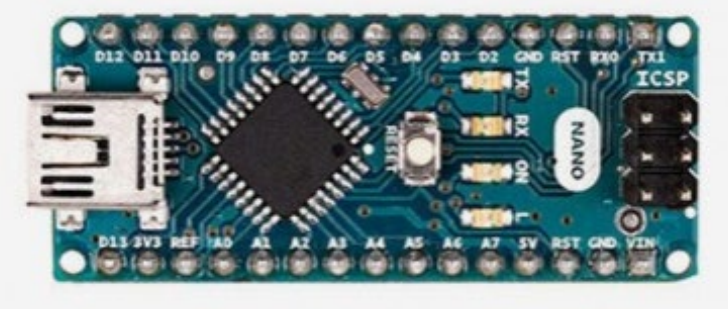

Fig.3. Arduino Nano board

\subsection{Data monitoring unit}

Fire alarm equipment with a single information source is prone to false alarms or untimely alarms. The system adopts infrared sensor and smoke sensor as the data monitoring unit of this system.

The infrared sensor can collect the light signal in the range of 750 1100 nanometers, and is very sensitive to the flame spectrum. It can judge whether the flame is detected by converting the light signal into a high and low-level signal by calculation. The detection angle of the sensor is about $60^{\circ}$, and the working voltage is $3.3 \mathrm{~V} \sim 5 \mathrm{~V}$.

The infrared sensor is shown in Fig.4.

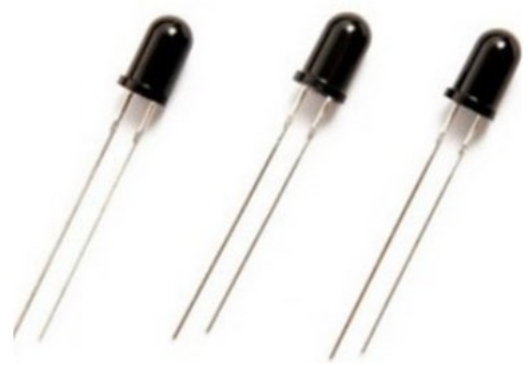

Fig.4. infrared sensor

In the early stage of a fire, a lot of smoke is generated, so the detection of smoke can also be used as one of the methods of fire monitoring. The system uses the MQ2 smoke sensor as the smoke data collection module. The sensor has a response time within $10 \mathrm{~s}$ and can monitor smoke data from 300PPM to 10000PPM.

The MQ2 smoke sensor is shown in Fig.5.

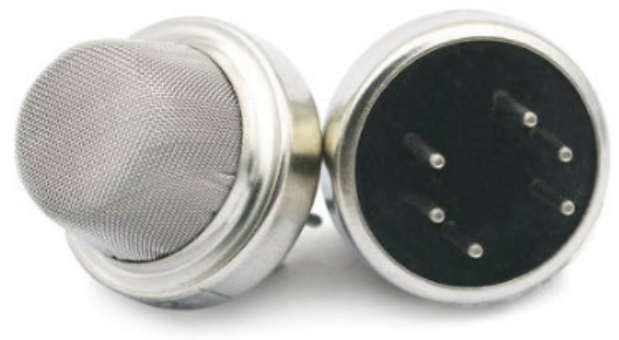

Fig.5. MQ2 smoke sensor

\subsection{Communication unit}

Aiming at the problem of complicated wiring of wired communication equipment and prone to failure, this system uses wireless communication for data exchange. For the communication between nodes, this system selects the CC2530 radio frequency module as the communication unit between nodes, which has the function of ZigBee wireless networking. This module has the following characteristics:

(C) The 2021 International Conference on Artificial Life and Robotics (ICAROB2021), January 21 to 24, 2021 
- With strong adaptability, nodes can be added or deleted according to the needs of the usage scenario

- Effective communication distance: $100 \mathrm{~m}$

- Low power consumption

After the information is summarized to the master node, the data will be sent to the client through the ESP8266 WIFI module to realize remote monitoring.

When a fire occurs, the system may face power outages and network outages. In order to send the alarm information to relevant persons in time, the system is equipped with a GPRS SIM300C communication module, which can carry out a fire alarm in the form of SMS notification.

\subsection{Guiding unit}

Multi-directional LED indicators and MP3 voice module constitute the system's guiding unit. Multi-directional LED indicator, the main body is composed of red and green LED lights. The MP3 voice module supports audio output in MP3 and WAV formats, which can be controlled through the serial port of the microcontroller.

The design of the sound and light evacuation module is shown in Fig.6.

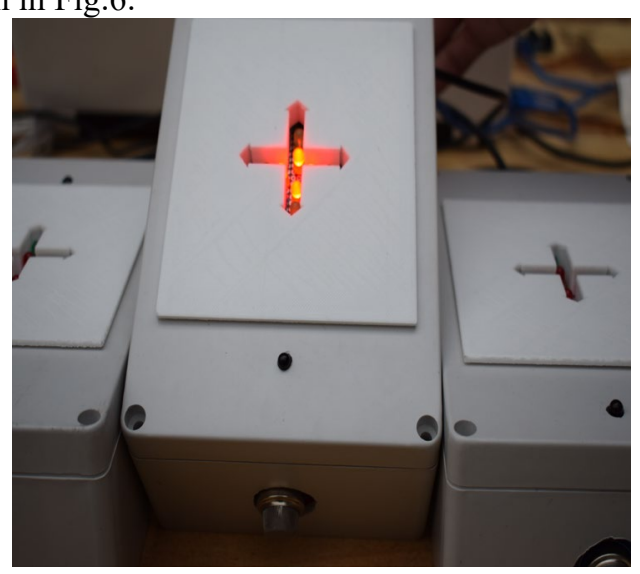

Fig.6. Sound and light evacuation module

\section{Introduction of functional module}

\subsection{Data monitoring function}

The system uses infrared sensors and smoke sensors to collect information and sends it to the client for digital display. The user can view the monitoring data, working status, and alarm status of each node through the system interface. The client is written in $\mathrm{C \#}$ language and has good compatibility and stability.
The design of the monitoring software is shown in Fig.7.

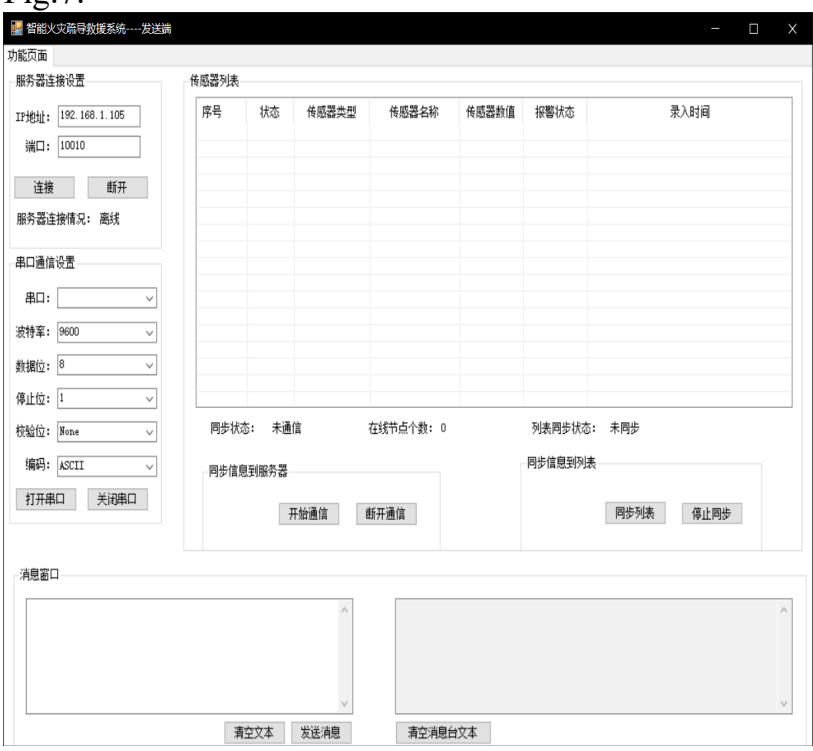

Fig.7. monitoring software

\subsection{Design of Hierarchical alarm function}

In order to prevent invalid alarms due to sensor failures or operating errors, this system has designed a hierarchical alarm function. The discrimination mechanism will divide the fire situation into two levels according to the number of alarm nodes and the data of alarm node.

When the fire is small and easy to extinguish, the system will send an alarm message to the corresponding person in charge to notify him to deal with it. When the fire situation is large or there is a continuous development trend, it will directly send an alarm message to the neighboring fire department through the alarm platform.

\subsection{Sound and light guiding function}

In order to effectively guide the crowd, the system has designed a light guide and voice prompt guide.

The light grooming function is completed by a multi-directional indicator, which will display red in the direction close to the fire point and green in the direction far away from the fire point according to the information of the alarm node.

(C) The 2021 International Conference on Artificial Life and Robotics (ICAROB2021), January 21 to 24, 2021 
Yuhui Cheng, Tianyi Zhang

The function of the voice prompt is realized by the voice module. When a fire breaks out, the system will broadcast an alarm and broadcast the approximate location of the fire based on the node location information entered in advance to provide guidance for crowd evacuation.

\section{Conclusion}

This fire alarm system is suitable for high-rise buildings or crowded indoor shopping malls. After a fire occurs, it can report to the police in time, and provide a reasonable evacuation route according to the location parameters of each fire detection node to facilitate the evacuation of the crowd.

\section{References}

1. Jianguo Wang, Baoyi Guo, Monitoring system based on ZigBee and WiFi buildings combining, Journal of Xi'an Technology University, 2008, 28(5): pp.460-465.

2. Fan Yang, Maolin Chen, Xun $\mathrm{Wu}$, et al., Forest fire alarm system based on sensor information fusion technology, Journal of Huazhong University of Science and Technology: Natural Science Edition, 2013, 41(2): pp.22-25. 